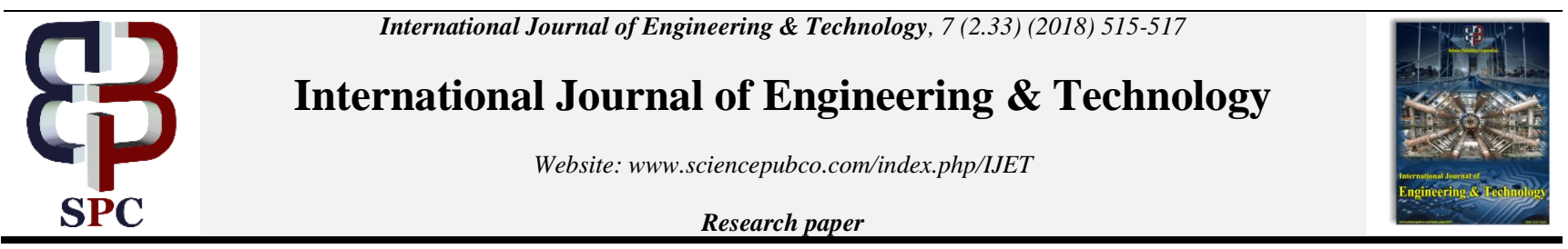

\title{
Seed based plant propagation algorithm for multiple travelling salesman problem
}

\author{
S. Kalaiarasi ${ }^{1} *$, P. Sriramya ${ }^{1}$ \\ ${ }^{1}$ Saveetha School of Engineering, Saveetha Institute of Medical and Technical Sciences, Chennai, India \\ *Corresponding author E-mail: sriramya82@gmail.com
}

\begin{abstract}
Multiple Travelling Salesman Problem is a complex problem in which route for a salesman is assigned to visit a city that has various hurdles such as congested road, damaged road, etc. In recent years biologically inspired algorithms are most widely used to solve many optimization problems. Here seed based plant propagation algorithm is applied for the multiple travelling salesman problem that is also a optimization problem, and the result is compared with a short-cut routing algorithm. The result shows that Seed based Propagation Algorithm is easy to implement since it has few parameters to be utilized and also time complexity is reduced when implemented in multiple travelling salesman problem.
\end{abstract}

Keywords: Multiple Travelling Salesman Problem; Seed Dispersal; Short Cut Routing; Plant Propagation Algorithm.

\section{Introduction}

The seed based plant propagation algorithm relies on the dispersion of seeds and propagation of plant in strawberry plant. Plants are the major part of environment. Most plants produce a large number of seeds which germinate and grow into new plants. A single plant produces many seeds. But not all seeds grow into new plants. They are (i) Some seeds are eaten up by birds, rats and other animals. (ii) Some of them may get destroyed by wind and rain. (iii) Some seeds do not grow because they do not get sufficient air, water or warmth. The scattering of seeds away from the mother plant is called dispersion of seeds. This seed based propagation algorithm inhibits the dispersion behavior of plant and also the propagating behavior of strawberry plant into new plant.

The plant propagation algorithm [1] works on the principle that plants with more nutrients used to send more number of short runners. The plant also sends less number of long runners when the nutrient level is low. The search space is explored by the long runners and the solution space is exploited by the short runners, [1].

This propagation behavior helps in assigning the shortest routes to the salesman in the multiple travelling salesman problem multiple travelling salesman problem.

\section{Multiple traveling salesman problem}

The multiple travelling salesman problem is applied for many real world applications. Real world applications may be computer wiring, overhauling gas turbine engine, order-picking problems etc [2] [3]. In multiple travelling salesman problem many factors has to be considered like vehicle condition used by the salesman, road condition. There may be pits in the road that causes the travel difficult. So waiting time gets increased. The main aim behind the multiple travelling salesman problem is to reduce the waiting time and the travel time.
In multiple travelling salesman problem ' $b$ ' salesman has to visit a collection of ' $k$ 'cities and each salesman will have the same origin and the destination place. The constraints are each salesman can visit each place one time. Different cases of multiple travelling salesman problem exist [4] [5]. They are multiple depots, single depots, etc. A route returning to the beginning is known as Hamiltonian Circuit [11]. A route note returning to the beginning is known as Hamiltonian path [11]. Vehicle routing is an extension of multiple travelling salesman problem. In vehicle routing, salesman meets customers within a stipulated time using vehicle that has limited capacity [12].

\section{Problem definition}

There are so many solution methods are available like trying every possibility, Optimization methods and heuristic methods. When trying all the possibility, suppose if it takes 1 microsecond to calculate each possibility takes 10140 centuries to calculate all possibilities when $n=100$. The optimizing methods obtain guaranteed optimal solution, but can take a very long time. But Heuristic methods obtain good solution quickly by intuitive methods. In this case there is no guarantee of optimality. The Seed based plant propagation algorithm is the optimizing method used to solve multiple travelling salesman problem. 


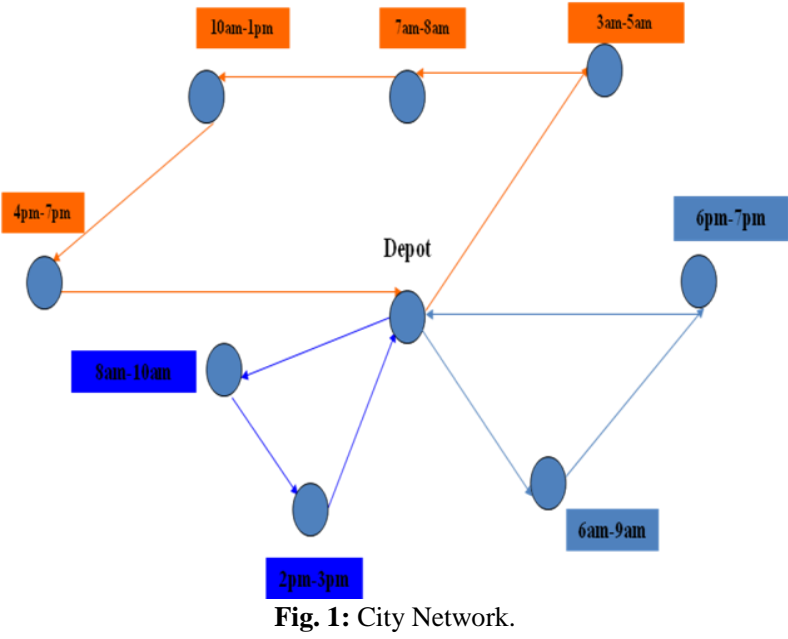

\subsection{Short-cut routing algorithm}

The short cut routing algorithm is used to determine the shortest path between the various nodes in a graph. It is calculated using the edges weight in the graph. The algorithm overcomes the drawback found in the Dijkstra's algorithm [13]. The main aim of this algorithm is to minimize the time complexity by using different levels. The various levels and types of vehicles are listed in Table 1 [10]. To these values SR algorithm is applied and the calculated time complexity is shown in table 2 [10]. The level one salesmen starts their journey from the city allocated using the vehicles assigned correspondingly. The algorithm uses the graph with vertices $V_{e}$ and the edges $E_{d}$ and the set of salesman that is $S_{1} l_{1}$, $\mathrm{Sal}_{2} \ldots$ Saln values are used. The SR Algorithm executes till the salesman travel through all the cities. The shortest path is found by the salesman along with the corresponding vehicle.

Table 1: Threshold Values Given as Input to SR Algorithm and Sbppa

\begin{tabular}{llll}
\hline Salesman & Level & Types of Vehicles & Vehicles \\
\hline 1 & 1 & V1 & 1 \\
4 & 2 & V2 & 4 \\
16 & 3 & V3 & 16 \\
64 & 4 & V4 & 64 \\
\hline
\end{tabular}

The Shortcut Routing Algorithm takes graph with vertices v, edges $\mathrm{E}$ and weight on edges as Wei. It takes the set of salesman to be Sal $[r]$ where $r=1,2, . . k$, and set of processor to be $\operatorname{Pr} o[r]$. The array of salesman is partitioned. Next for each salesman values are provided to the processor Pro[r] and then adjacency matrix $\mathrm{T}$ values are found. Then the vertex $\mathrm{v}$ is read and the distance value dista [] is initialized with value ' 0 '. Then short distance is found based upon the value of $\mathrm{T}$ and with the help of below given formula

Shpa $=\operatorname{dista}[\mathrm{c}]+$ dista-between $(\mathrm{c}, \mathrm{v})$;

Then shpa value and the dista value is compared. If the shpa value is less than the dista value, that value is assigned to dista [].

\subsection{Seed based plant propagation algorithm}

The Seed based plant propagation algorithm SbPPA is applied for many constraints and unconstraint problem and the results shows that this algorithm converges very faster than any other naturally inspired algorithms. In this algorithm, the population is selected randomly. And also number of trail runs is also selected. Next the best solutions are taken from trail run. It is important to balance exploration and exploitation. So a threshold value is chosen to find to which extent exploration and exploitation has to be done. It is done using poisson probability and if probability value is $<0.05$ it implies that exploitation is complete.

The SbPPA has the following steps [1]:
Step 1: Create a random population of seeds pop $=\{\mathrm{Xi} \mid \mathrm{i}=1$, $2, . . \mathrm{NP}\}$ and retrieve the optimal solution from each iteration. popbest.

Step 2: The dispersal of seeds in the neighborhood of the SPs is done by both, ripe fruits falling from strawberry plants or by agents. The step length is calculated.

Step 3: Seeds are spread globally through agents. The step lengths for these travelling agents are drawn from the Levy distribution [7].

Step 4: The probabilities, Poiss $(\lambda)$, a balancing factor, that is calculated between exploration and exploitation.

Let NP be the number of salesman involved in the multiple Travelling Salesman Problem. According to the SBPPA arrival of multiple salesman to the place is found using poisson distribution [6]. The mean arrival rate of multiple salesman to the places is found using levys distribution [7] [8]. It is essential for this algorithm to balance exploration and exploitation. So a threshold value is chosen to find to which extent exploration and exploitation has to be done. It is done using poisson probability and if probability value is $<0.05$ it implies that exploitation is complete.

\subsection{Experimentation and discussion}

In this paper, the Seed-Based Plant Propagation Algorithm (SbPPA) [1] is applied to the multiple travelling salesman problem. Plants evolved in a variety of ways to propagate. The strawberry plant uses runners and seeds to propagate. The SbPPA is applied to multiple travelling salesman problem. It uses single processor for its execution and the time taken to execute the algorithm for different number are nodes are calculated.

The time complexity of the SbPPA is found by finding the time taken to execute the algorithm on the given number of inputs. The best solutions taken from the each trial run is given as input to the algorithm. To find the global and local dispersion of seeds, poisson distribution is used. It is the constant value so the running time will not change in relation to N. For each and every input the algorithm is applied. So the complexity will be ' $\mathrm{n}$ ' for $\mathrm{n}$ input that is the population size. And also algorithm is applied for ' $n$ ' dimension range. The running time is shown in table III. The running time for the SR algorithm for multiple travelling salesman problem is shown in table II [10]. The time complexity of SbPPd on multiple travelling salesman problem is $\mathrm{O}(\mathrm{n} 2)$. It is compared with time taken by short-cut routing algorithm [10], which is $\mathrm{O}$ (n3) [10].

Table 2: Complexity Using Short Cut Routing Algorithm

\begin{tabular}{lll}
\hline Processor & Nodes & Time Taken \\
\hline 1 & 4 & 64 \\
1 & 12 & 432 \\
1 & 48 & 6912 \\
1 & 192 & 117649 \\
\hline
\end{tabular}

Table 1: Complexity Using SbPPA

\begin{tabular}{lll}
\hline Processor & Nodes & Time Taken \\
\hline 1 & 4 & 64 \\
1 & 12 & 231 \\
1 & 48 & 3521 \\
1 & 192 & 45275 \\
\hline
\end{tabular}

\section{Conclusion}

The result is represented in the line graph and it is shown in fig. 2 . The line is plotted in the graph with number of nodes at horizontal axis and the time taken at vertical axis. By looking at the line it is known that the computational complexity of SbPPA is lesser when compared with computational complexity of the short-cut routing Algorithm. The time complexity of short-cut routing algorithm is $\mathrm{O}$ (n3) [10]. Here the complexity of SbPPA is calculated to be O (n2). SbPPA is easy to implement because it requires less arbitrary parameter settings when compared to any other algorithms. So the computational complexity is lesser. 


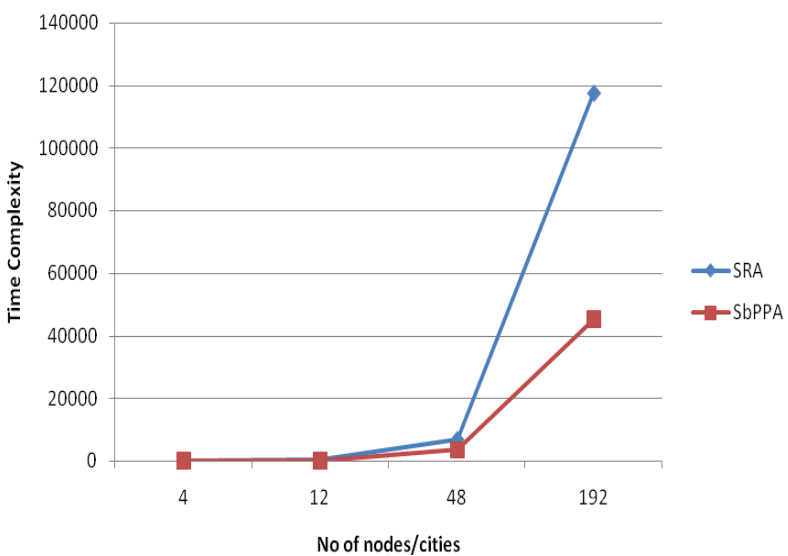

Fig. 2: Performance of SR and SbPPA.

\section{References}

[1] Muhammad Sulaiman, Abdellah Salhi1, "A Seed-Based Plant Propagation Algorithm: The Feeding Station Model," Scientific World Journal, Hindawi Publishing Corporation, Volume 2015, Article ID 904364.

[2] Andras Kiraly and Janos Abonyi, "Optimization of Multiple Traveling Salesmen Problem by a Novel Representation Based Genetic Algorithm", Intelligent Computational Optimization in Engineering Studies in Computational Intelligence Vol. 366, pp. 241-269, 2011.

[3] Paul Oberli, Sivakumar Rathinam and Swaroop Darbha, "A Transformation for a Multiple Depot, Multiple Traveling Salesman Problem." American Control Conference Hyatt Regency Riverfront, St. Louis, MO, USA June 10-12, 2009.

[4] Abraham P. Punnen. "The Traveling Salesman Problem and Its Variations".

[5] Sai Yadlapalli, Sivakumar Rathinam and Swaroop Darbha, "3Approximation algorithm for a two depot, heterogeneous traveling salesman problem", Vol. 6, No. 1, pp. 141-152, Jan 2012.

[6] J. A. Lawrence and B. A. Pasternack, Applied Management Science,Wiley, New York, NY, USA, 2002.

[7] M. Sulaiman and A. Salhi, "A seed-based plant propagation algorithm: the feeding station model," in Proceedings of the 5th International Conference on Metaheuristics and Nature Inspired Computing, Marrakesh, Morocco, October 2014.

[8] X.-S. Yang, Nature-Inspired Metaheuristic Algorithms, Luniver Press, Beckington, UK, 2011.

[9] Gustavo Marfia, David Mack and Giovanni Pau, Cecilia Mascolo "On the Effectiveness of an Opportunistic Traffic Management System for Vehicular Networks", Intelligent Transportation Systems, IEEE Transaction, Vol. 12, No. 4, December 2012.

[10] Kalaiarasi, P. Sriramya and R. A. Karthika, "A comparative study of sequential algorithm and shortcut routing algorithm on multiple travelling salesman problem", ARPN Journal of Engineering and Applied Sciences, vol. 10, No 13, July 2015

[11] Csehi, C. Gy. and Toth, J, "Search for Hamiltonian Cycles," Mathematica J. 13, 2011.

[12] Asaf Levin and Uri Yovel, "Local search algorithms for multipledepot vehicle routing andfor multiple traveling salesman problems with proved performance guarantees," December, 2012.

[13] Gintaras Vaira and Olga Kurasova, "Parallel Bidirectional Dijkstra's Shortest Path Algorithm," Proceedings of the 2011 Conference on Databases and Information Systems VI", 2011. 